\title{
The characterization of a pressure sensor constructed from a knitted spacer structure
}

\author{
Theodore Hughes-Riley ${ }^{*}{ }^{\text {, Carlos Oliveira }}{ }^{1}$, Robert H. Morris ${ }^{2}$, Tilak Dias ${ }^{1}$ \\ ${ }^{1}$ Advanced Textiles Research Group, School of Art and Design, Nottingham Trent University, ${ }^{2}$ Department of Physics and \\ Mathematics, School of Science and Technology, Nottingham Trent University, Nottingham, United Kingdom
}

\begin{abstract}
Background and Objectives: This study investigates a novel type of textile pressure sensor fabricated in a single production step. The work characterizes two designs of electronic textile pressure sensor creating new knowledge into the operation of these types of textile sensors. Interest in electronic flexible film and electronic textile pressure sensing has grown in recent years given their potential in medical applications, principally in developing monitoring solutions for wheelchair users and hospital patients to help prevent the formation of pressure ulcers. Materials and Methods: Two designs of textile pressure sensor were produced using computerized flat-bed knitting. One design was produced in a single step, where the conductive tracks were incorporated into the top and bottom surfaces of a knitted spacer structure (knitted spacer pressure sensor). The other sensor was comprised of separate knitted layers. The response of the sensors was tested by changing the applied pressure in two ways: By altering the applied force or changing the area of the applied force. Sensor hysteresis and how the sensor thickness affected its response were also examined. Results: The two sensor designs behaved differently under the tested conditions. The knitted spacer pressure sensor was pressure sensitive up to $25 \mathrm{kPa}$ and showed no hysteretic effects over the pressure range of interest. Conclusions: This study presents a fully textile pressure sensor that was produced with a single production step and demonstrates its functionality over the pressure range of interest for monitoring wheelchair users.
\end{abstract}

Keywords: Electronic textiles, e-textiles, pressure sensor, pressure ulcers, smart textiles

\section{INTRODUCTION}

This work presents a novel textile pressure sensor concept, where an electronically functional textile pressure sensor was created using a single-step manufacturing process. The sensor is fully textile and did not make use of lamination or printing methods. Further, the work investigated the correct methodology for testing this type of textile sensor. The pressure

\begin{tabular}{l}
\hline *Address for correspondence: \\
Dr. Theodore Hughes-Riley, Advanced Textiles Research \\
Group, School of Art and Design, Nottingham Trent University, \\
Nottingham, United Kingdom. \\
E-mail: theodore.hughesriley@ntu.ac.uk \\
\hline \multicolumn{2}{c|}{ Access this article online } \\
\hline \multicolumn{2}{|c|}{ Quick Response Code } & Website: \\
\end{tabular}

sensor was developed to help combat the formation of pressure ulcers.

Ulceration in hospital patients and wheelchair users is a serious problem, with pressure ulcers currently costing the UK National Health Service (NHS) between $\leq 1.4$ and $\leq 2.1$ billion a year (4\% of the NHS budget) ${ }^{[1]}$ Pressure ulcers form when soft tissues and the skin are in prolonged contact with a surface, as the pressure reduces the blood flow, resulting in cell death. To prevent the formation of ulcers, it is advisable for the weight of the person to be repositioned frequently. Currently, in a hospital setting, this is achieved

This is an open access journal, and articles are distributed under the terms of the Creative Commons Attribution-NonCommercial-ShareAlike 4.0 License, which allows others to remix, tweak, and build upon the work non-commercially, as long as appropriate credit is given and the new creations are licensed under the identical terms.

For reprints contact: reprints@ @medknow.com

How to cite this article: Hughes-Riley T, Oliveira C, Morris RH, Dias T. The characterization of a pressure sensor constructed from a knitted spacer structure. Digit Med 0;0:0. 
Hughes-Riley, et al.: A knitted spacer pressure sensor

by moving prone patients once every $2 \mathrm{~h}$; however, in some cases, this is not possible given the time constraints on medical staff. For a wheelchair user, this is more difficult; users that cannot reposition themselves will be more likely to develop ulcers, as it is recommended that they reposition once every $15 \mathrm{~min} \cdot{ }^{[2]}$ Subsequently, it is desirable to develop a method to monitor the pressure applied at different points on a seat or bedding (i.e., on a wheelchair or bed). With such a technique if pressure is applied for a long time, a caregiver or the end user can be informed and repositioning can be carried out as a preventative measure.

A variety of pressure sensing modalities exist and have been utilized for physiological measurements. ${ }^{[3-5]}$ While these systems may provide highly accurate pressure measurement, they may not be comfortable for the end user to lay on or sit on. In the case of some systems, such as film-based electronic sensors, the sensor might bend, but these will not conform to the body, making it noticeable to the end user.

Textiles provide a basis for making human pressure measurements, as they are both comfortable, conformal, and breathable. As a result, there has been significantinterest in recent years into the development of electronic textile pressure sensors. ${ }^{[5-12]}$ There are a variety of commercially available pressure sensing products that are both thin and flexible; however, many details are not forthcoming and it is unclear whether these are true textiles or flexible film-based systems. ${ }^{[13,14]}$ Most electronic and electronic textile pressure sensors use either a capacitive or resistive measurement, where the application of pressure effects one of these two properties. For nontextile capacitive pressure sensors, there are certain reported advantages in a medical situation, as they show less sensitivity to temperature changes and humidity. Capacitive sensors have also been reported to provide an average pressure load over their surface, while resistive sensors provide a peak pressure value. ${ }^{[7]}$ The academic literature has largely focused on capacitance-based textile pressure sensing. ${ }^{[5-12]}$ An overview of electronic textile pressure sensors is available elsewhere. ${ }^{[13,14]}$

The basic construction of most textile pressure sensors consists of two fabric layers sandwiching a pressure sensitive material, which is made of either a dielectric or a highly electrically resistive material. The two fabric layers contain electrically conductive electrodes crafted by either printing a conductive ink on to the fabrics or by weaving or knitting conductive yarns. ${ }^{[6,13,14]}$ As the fabric is deformed by the application of pressure, the electrodes are brought closer to one another, changing the capacitance or the resistance recorded.

The aim of this study is to present an innovative electronically functional textile pressure sensor where the capacitance of the sensor changes with the application of pressure. The sensor is constructed from a single-layer knitted spacer structure produced using the state-of-the-art computerized flat-bed knitting technology. The knitted spacer is a three-dimensional (3D) structure consisting of two outer surfaces joined by a central fibrous structure.

During the manufacturing, process electrodes were incorporated on to the two outer surfaces of the spacer structure using the intarsia technique with silver-plated nylon yarn and with polyester fibers between the surfaces acting as a dielectric layer. This sensor is referred to as the knitted spacer pressure sensor throughout this publication. Rectangular electrodes were formed, with the top electrodes and bottom electrodes perpendicular to one another creating an addressable grid of discrete junctions where the electrodes overlap.

This novel approach offers significant advantages over alternative designs as producing the pressure sensor in this manner would ensure that the electrodes were fixed in place and would not move relative to one another, improving resilience. This also substantially simplifies the manufacturing process of the sensor, reducing its cost, and increasing its commercial viability, which is essential for widespread adoption.

It is also highly important to correctly characterize the behavior of these sensors when pressure is applied; changing pressure by either altering the applied force or the area over which the force is applied must be considered. The methodology used to characterize other pressure sensor types (i.e., thin films, bladder based pressure sensors, etc.) may not be valid in a textile setting. The flex and shear properties of a textile mean that the deformation of the material will alter the shape of the textile away from the point where the pressure is applied. Therefore, deformation of the textile away from the point where the electrodes intersect may or may not influence results. Similarly, the structure of the knitted spacer structure will have an influence on the deformation of the textile. The complex deformation of a textile structure is difficult to accurately model, and therefore, this behavior has to be studied experimentally.

To better characterize these effects and to understand how the knitted spacer pressure sensor's construction 
Hughes-Riley, et al.: A knitted spacer pressure sensor

could affect its performance, a second capacitive sensor design was employed, where a textile pocket of separate textile electrodes was produced where a spacer structure could be placed inside, referred to as the knitted pocket pressure sensor in this publication. This created a pressure sensor with three discrete layers, which more closely represent other contemporary capacitive fabric sensors such as those by Meyer et al. ${ }^{[8,11]}$ or Hoffmann et al. ${ }^{[15]}$ who use multiple separate layers to create capacitive textile pressure sensors.

The design requirements of the pressure sensors developed in this study were informed by the literature. The focus was to create a system suitable for wheelchair users in the first instance; hence, a minimum junction size of $14 \mathrm{~mm} \times 14 \mathrm{~mm}$ was chosen, based on the maximum suitable diameter of a pressure sensing element identified in the literature. ${ }^{[16]}$ There is a wealth of literature that discuss the pressure at the seat-body interface; however, work by Hobson ${ }^{[17]}$ has been used to determine the maximum pressure of interest in this study into the knitted spacer pressure sensor. Hobson's work compared data from nondisabled individuals and individuals with spinal cord injuries, observing higher maximum pressures at the interface for the latter group. This group would be more representative of the intended users for developing a wheelchair pressure monitoring solution. A maximum pressure of around $190 \mathrm{mmHg}(25.3 \mathrm{kPa})$ was presented in Hobson's work, and therefore, an operational pressure range covering $0-25 \mathrm{kPa}$ was desirable for the characterization of the knitted spacer pressure sensor. This pressure range would also be suitable for monitoring the pressure applied by a patient lying prone in a bed for long period of time, where the range of interest is up to $7.7 \mathrm{kPa} \cdot{ }^{[18]}$ The sensor would have to be capable of taking readings at intervals on the order of minutes, given the time frame that ulcers form over. As a result, the step-response time of the sensor was not investigated as part of this study.

In this study, two textile pressure sensors (knitted spacer pressure sensor and knitted pocket pressure sensor) were produced and then characterized by changing the applied pressure by both altering the applied force and by changing the area over which force was applied. This allowed the response of the textile sensor to be fully understood over a range of circumstances that it might be subjected to operationally.

The hysteresis of the produced textile pressure sensors was examined by incrementally applying and then removing force and examining the sensor response. Hysteresis is a known problem for textile sensors presented in the literature..$^{[1]}$

Finally, additional experiments were conducted to optimize the design of the knitted spacer pressure sensor. There are three major factors that will affect the sensors' performance such as the electrode size, the dielectric properties of the material between the electrodes, and the electrode separation. The electrode size was informed by the literature and could not be made significantly larger without the sensor losing the necessary spatial resolution, and therefore, electrode size was not explored in this study. The dielectric properties of the material between the electrodes, in this case, polyester, would potentially be influenced by moisture content; however, it was assumed that the very low moisture absorbency of polyester meant that moisture content was unlikely to influence results. The separation of the electrodes was investigated, and two thinner knitted spacer pressure sensors were produced and characterized.

This work has generated the fundamental scientific base necessary to create a textile pressure sensor as a single fabric layer and manufactured in a one-stage process. This will allow for the creation of a larger pressure sensor suitable for use by wheelchair users and for hospital bedding in the future; however, the development of this system was beyond the scope of this study.

\section{MATERIALS AND METHODS}

\section{Pressure sensor design and fabrication}

Two distinct types of pressure sensor were presented in this work. Both sensors were $170 \mathrm{~mm} \times 155 \mathrm{~mm}$ and included five complete $12-\mathrm{mm}$ wide knitted silver conductive electrodes (conductive yarn: Shieldex ${ }^{\circledR}$ 235/32 dtex-2-ply-HC +B silver yarn, Shieldex ${ }^{\circledR}$, Bremen, Germany) on either surface. The electrodes were separated by $12 \mathrm{~mm}$, and the electrodes on the top of the sensors were perpendicular to those on the bottom creating a $2 \mathrm{D}$ grid of junctions [Figure 1]. The samples were produced using a Stoll ADF 3 E14 fully computerized flat-bed knitting machine (Stoll, Reutlingen, Germany). The nonconductive threads used in the samples were made from polyester yarn (164/48 dtex).

Two variations of pressure sensors were produced: one made in a single stage (knitted spacer pressure sensor) and one produced as a pocket in which a spacer fabric could be inserted (knitted pocket pressure sensor). The knitted spacer pressure sensor was produced in a single step and was approximately $3.2-\mathrm{mm}$ thick. The knitted 
Hughes-Riley, et al.: A knitted spacer pressure sensor

pocket pressure sensor had a space to insert a separated 2.9-mm thick knitted spacer fabric giving the final sensor a thickness of approximately $3.2 \mathrm{~mm}$.

Additional optimization experiments were conducted for the knitted spacer pressure sensor. Two additional knitted spacer pressure sensor were produced, one with a thickness of $2.6 \mathrm{~mm}$ and one with a thickness of $2.8 \mathrm{~mm}$. Ultimately, for the comfort of the end user, it was desirable to create a pressure sensor that was as thin as possible. For clarity, Table 1 shows the characteristics of each of the samples explored in this work.

\section{Pressure sensor testing procedure}

Textile fabrics are soft materials and do not behave in the same way as rigid structures or even thin films, making any assumptions about how a textile capacitor would behave to changing the force applied to it, or the area of the applied force naïve. This is particularly true for thicker structures, such as those described in this work, as a thicker material is more likely to deform in complex ways; therefore, pressure changes due to both changes in force and changes in the area over which force was applied needed to be understood, as the result would not necessarily be identical.

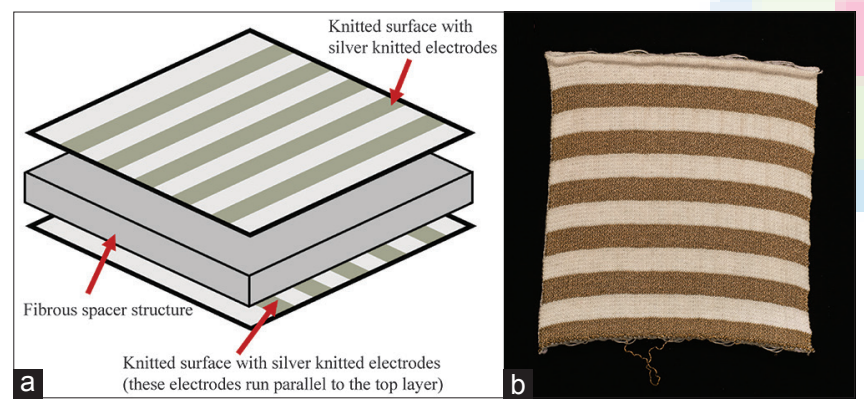

Figure 1: Knitted spacer pressure sensor. (a) Schematic of the knitted spacer pressure sensor. The upper and lower surfaces of the spacer fabric had knitted conductive silver electrodes, with the electrodes perpendicular to one another to create a grid of discrete junctions. The upper and lower fabric surfaces were separated by a fibrous middle layer. (b) Photograph of the knitted spacer pressure sensor from above. The surface of the knitted spacer pressure sensor and outer layer of the knitted pocket pressure sensor look identical. Note that there is an incomplete sixth track at the bottom of the sensor
The pressure sensors were tested using round bespoke acrylic disks (15-70-mm diameter) and weights (cast zinc alloy and cast iron, Breckland Scientific, Liverpool, UK). This allowed for either the force or the area of the applied force to be altered in a repeatable way. In the data presented, the central junction of the pressure sensors was used [i.e., at the center of the sensor, Figure 1b].

For experiments where area was changed, seven disks with diameters ranging between 15 and $70 \mathrm{~mm}$, with a corresponding area of $1.8 \times 10^{-4} \mathrm{~m}^{2}-3.8 \times 10^{-3} \mathrm{~m}^{2}$, were used. A fixed force of $9.8 \mathrm{~N}$ was applied for each experiment giving an overall pressure range of $2.5-55.5 \mathrm{kPa}$.

Experiments exploring the effect of the applied force on the sensors' response covered 0.1-29.4 N, which corresponded to a $1.4-41.6 \mathrm{kPa}$ pressure change. For these experiments, the force was applied over an area of $7.1 \times 10^{-4} \mathrm{~m}^{2}$ (as a comparison, the sensor had a junction size of $\left.1.4 \times 10^{-4} \mathrm{~m}^{2}\right)$.

The effect of hysteresis on the sensors' response was also investigated. A range of forces (1.0-23.4 N) was applied to a $7.1 \times 10^{-4} \mathrm{~m}^{2}$ area resulting in a pressure range of $1.4-41.6 \mathrm{kPa}$. Pressure was applied incrementally and then removed showing the effect of both increasing and decreasing the pressure.

All capacitance values were measured using an Agilent 4192A LF impedance analyzer (Agilent Technologies, Santa Clara, USA), with readings taken using a $100 \mathrm{kHz}$ signal. Values were recorded once the capacitance readings had stabilized; it was observed that the capacitance readings stabilized 10-20 s after the pressure was changed. Values in this work are presented as a difference in capacitance; this being the capacitance recorded minus the capacitance value when no pressure was applied. The capacitance value when no pressure was applied was taken before each set of experiments.

Table 1: Summary of the pressure sensor characteristics

\begin{tabular}{lcl}
\hline & $\begin{array}{c}\text { Dimensions (thickness } \times \\
\text { width } \times \text { length), mm }\end{array}$ & Construction \\
\hline $\begin{array}{l}\text { Knitted pocket pressure } \\
\text { sensor }\end{array}$ & $3.2 \times 170.0 \times 155.0$ & $\begin{array}{l}\text { Knitted pocket structure with conductive electrodes } \\
\text { on either side and a removable knitted spacer fabric } \\
\text { Knitted spacer pressure }\end{array}$ \\
$\begin{array}{l}\text { Knitted spacer with conductive electrodes on either } \\
\text { sensor }\end{array}$ & $3.2 \times 170.0 \times 155.0$ & $\begin{array}{l}\text { side. Produced in a single step } \\
\text { Knitted spacer pressure } \\
\text { sensor }(2.6 \mathrm{~mm})\end{array}$ \\
$\begin{array}{l}\text { Knitted spacer pressure } \\
\text { sensor }(2.8 \mathrm{~mm})\end{array}$ & $2.6 \times 170.0 \times 155.0$ & $\begin{array}{l}\text { side. Produced in a single step } \\
\text { Knitted spacer with conductive electrodes on either } \\
\text { side. Produced in a single step }\end{array}$ \\
\hline
\end{tabular}


Hughes-Riley, et al.: A knitted spacer pressure sensor

Samples were tested under normal laboratory conditions. Changes in humidity were not believed to have an effect on the results given the low moisture absorbency of polyester fibers.

All experiments were conducted four times, and the standard deviation in the results was used to provide an estimation of the experimental error. The graphs in this work were produced using IGOR Pro (Version 7.0.2.2; Wavemetrics, Tigard, USA).

\section{RESULTS}

\section{Pressure sensor characterization}

The knitted pocket pressure sensor was subjected to different pressures with pressures varied by either changing the applied force or the area of the applied force. A range of pressures between 0 and $56 \mathrm{kPa}$ was explored. The results are shown in Figure 2a. Identical experiments were conducted on the knitted spacer pressure sensors as shown in Figure 2b.

Figure 2 clearly highlights the importance of fully characterizing and understanding the behavior of textile pressure sensors: Different pressures through changing either the force or area of applied force elicited different responses depending on the sensors' design. Figure 2a clearly showed that the knitted pocket pressure sensor was not pressure sensitive, with its response being a complex relationship between the applied force and the area over which force was applied.

By contrast, the knitted spacer pressure sensor was pressure sensitive, with the change in capacitance inversely related to the applied pressure regardless of whether the pressure change was due to a change in force or the area of the applied force. For both experiments, the data are in close agreement below $25 \mathrm{kPa}$. As a comparison, there was a $\sim 20 \%$ measurement difference at $\sim 30 \mathrm{kPa}$. Despite variations in the actual values recorded, under both conditions, the capacitance readings increased which would provide a suitable indicator of the application of pressure.

The difference between the two sensor designs is likely due to the multilayer structure of the knitted pocket pressure sensor as gaps can form between the electrode layers and central spacer structure: This may result in the different layers deforming differently under different conditions. This would not occur for the knitted spacer pressure sensor as the structure only comprised a single layer.

The hysteretic behavior of the two sensors was subsequently examined. Pressure was increasingly applied to each sensor in discrete steps up to $42 \mathrm{kPa}$, with capacitance measured at each point. The pressure was then reduced back to $0 \mathrm{kPa}$ in the same discrete steps, with the capacitance measured at each increment. Figure $3 \mathrm{a}$ shows the hysteretic behavior of the knitted pocket pressure sensor when pressure was applied and removed, with Figure 3b showing the same data for the knitted spacer pressure sensor.

The knitted pocket pressure sensor [Figure 3a] showed notable hysteresis beyond the experimental error; at $27.8 \mathrm{kPa}$, the change in capacitance was $320 \%$ larger
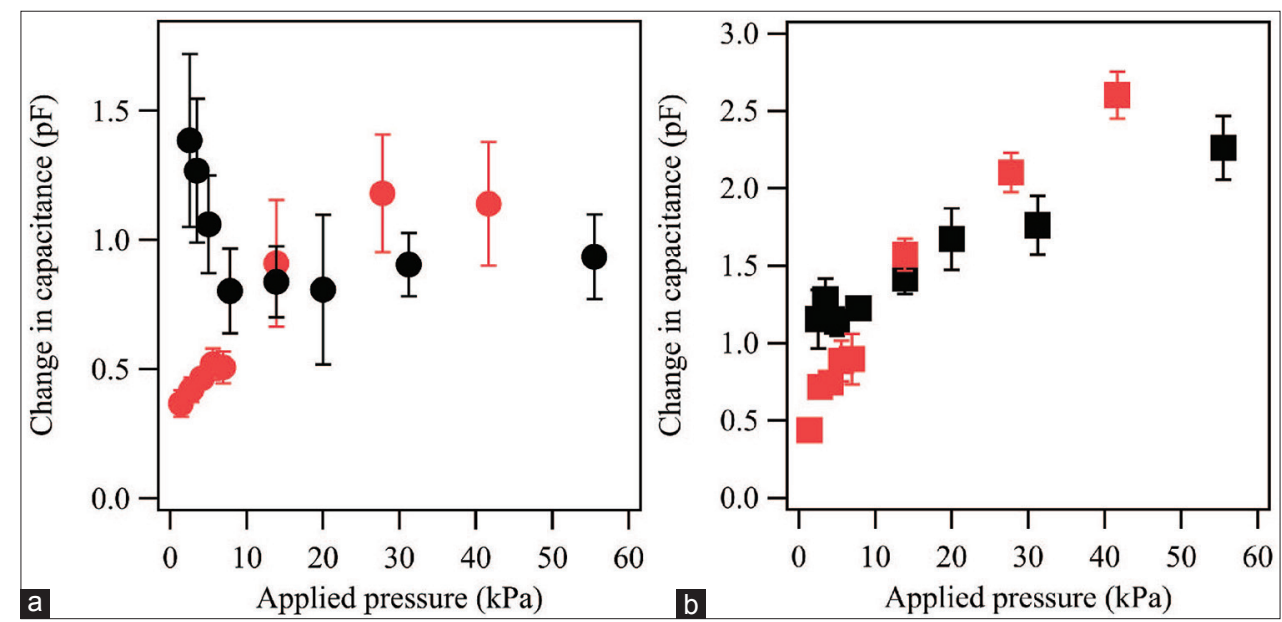

Figure 2: Knitted pressure sensors tested with different pressures. Pressure was changed by either changing the area of applied force (black) or the applied force (red). (a) Data collected using the knitted pocket pressure sensor. The sensor was sensitive to changes in the applied force ( $\bullet$ ) and changes in the area over which force was applied $(\bullet)$; however, in both cases, the responses were different making extracting meaningful pressure data problematic. (b) Pressure against the change in capacitance collected using the knitted spacer pressure sensor. Below $25 \mathrm{kPa}$, the sensor gave a similar response regardless of whether pressure was changed by altering the force $(\square)$ or the area that the force was applied to (घ). The relationship was similar regardless of whether force or area of applied force was changed 
Hughes-Riley, et al.: A knitted spacer pressure sensor

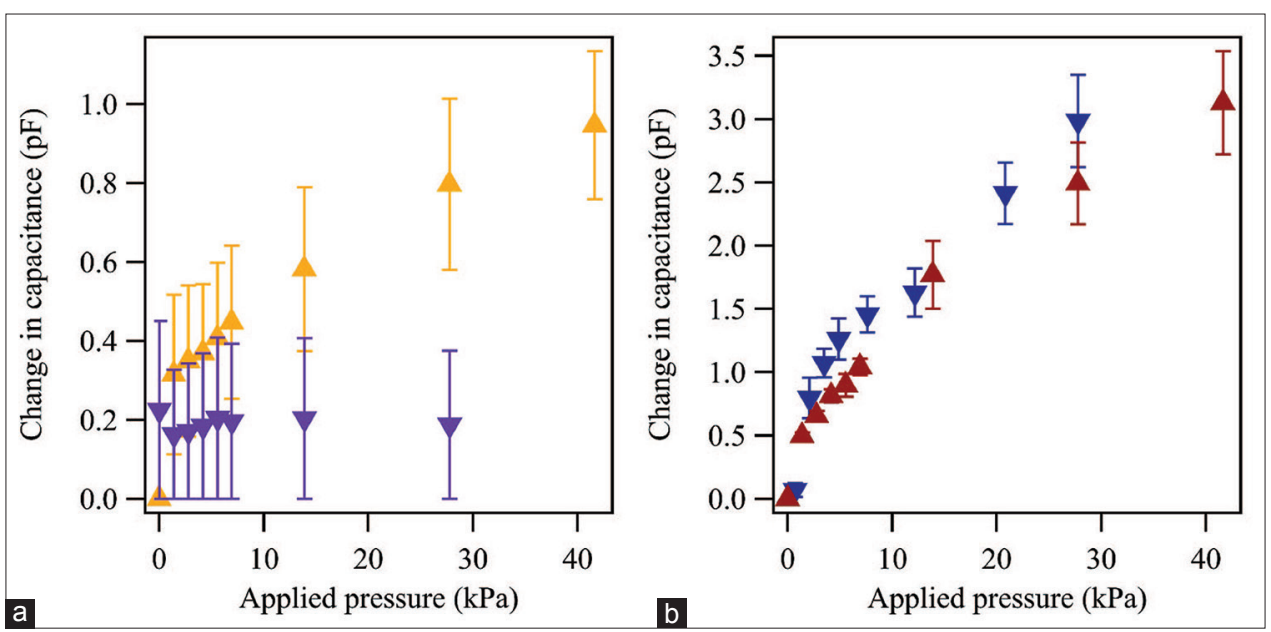

Figure 3: An analysis of the hysteresis for the two knitted pressure sensors. Pressure was gradually increased ( $\mathbf{\Delta}, \mathbf{\Delta})$ and then decreased $(\boldsymbol{\nabla}, \boldsymbol{\nabla})$. (a) The knitted pocket pressure sensor with $(\boldsymbol{\Lambda}, \boldsymbol{\nabla})$. (b) The knitted pressure sensor $(\boldsymbol{\Lambda}, \boldsymbol{\nabla})$. Unlike designs shown in the literature, the knitted pressure sensor overcame hysteretic effects, likely due to it comprising of only a single layer

when pressure was applied compared to when it was removed. This hysteresis was in line with results for similar multilayer capacitive sensors presented in the literature. ${ }^{[1]}$

Within the pressure range of interest, the knitted pressure sensor did not experience significant hysteretic effects, providing similar difference in capacitance results regardless of whether pressure was applied or removed. This was likely due to it comprising a single-layer knitted structure. In a system with multiple layers deformation between the layers can occur; for example, an air gap between the outer layer and spacer could form. This would not happen for the knitted pressure sensor as the entire material would change shape.

\section{Knitted spacer pressure sensor optimization}

Additional experiments were conducted to optimize the design of the knitted spacer pressure sensor, where two additional thicknesses of knitted spacer pressure sensor were produced: 2.6 and $2.8 \mathrm{~mm}$ (the original knitted spacer pressure sensor was $3.2 \mathrm{~mm}$ ). Thicker sensors were not produced as it was desirable to have a pressure sensor that was as thin as possible to maximize the comfort of the end user. A thinner sensor with a smaller separation between the top and bottom electrodes would also give the largest change in capacitance with pressure changes. Sensors thinner than $2.6 \mathrm{~mm}$ or thicker than $3.2 \mathrm{~mm}$ were not produced given the technical limitations of the flat-bed knitting machines.

Figure 4 shows the response of both sensors as a function of applied pressure.
The 2.6-mm thick knitted spacer pressure sensor was only sensitive to pressure changes up to $7.8 \mathrm{kPa}$, making it unsuitable for monitoring pressure for wheelchair users. At higher pressures, there was electrical contact between the upper and lower electrodes, preventing the sensor from operating correctly. Pressure changes induced by either changing the applied force or the area over which the force was applied gave similar but not identical results.

The 2.8-mm thick knitted spacer pressure sensor was consistently sensitive to changes in pressure up to 20 $\mathrm{kPa}$. While the sensor operated correctly up to 41.6 $\mathrm{kPa}$ when the applied force was changed, applying a force of $9.8 \mathrm{~N}$ over a smaller area $\left(3.1 \times 10^{-4} \mathrm{~m}^{2}\right.$ and $1.8 \times 10^{-4} \mathrm{~m}^{2}$, or 31.2 and $55.5 \mathrm{kPa}$, respectively) produced inconsistent results, with the sensor not consistently operating correctly for all experiments [as a note only measurements where four consecutive repeats were obtained are shown in Figure 4b]. This inconsistency is likely due to loose silver fibers becoming dislodged from the conductive yarn and creating electrical contact between the electrodes on either surface.

Of the three knitted spacer pressure sensors tested, only the 3.2-mm thickness sensor was suitable for monitoring the pressure applied by wheelchair users, as it was the only sensor design that could consistently measure pressures exceeding $25 \mathrm{kPa}$.

\section{Summary of the pressure sensor designs tested}

For clarity, the operating limitations of each of the four sensor designs explored in this work have been detailed 
Hughes-Riley, et al.: A knitted spacer pressure sensor

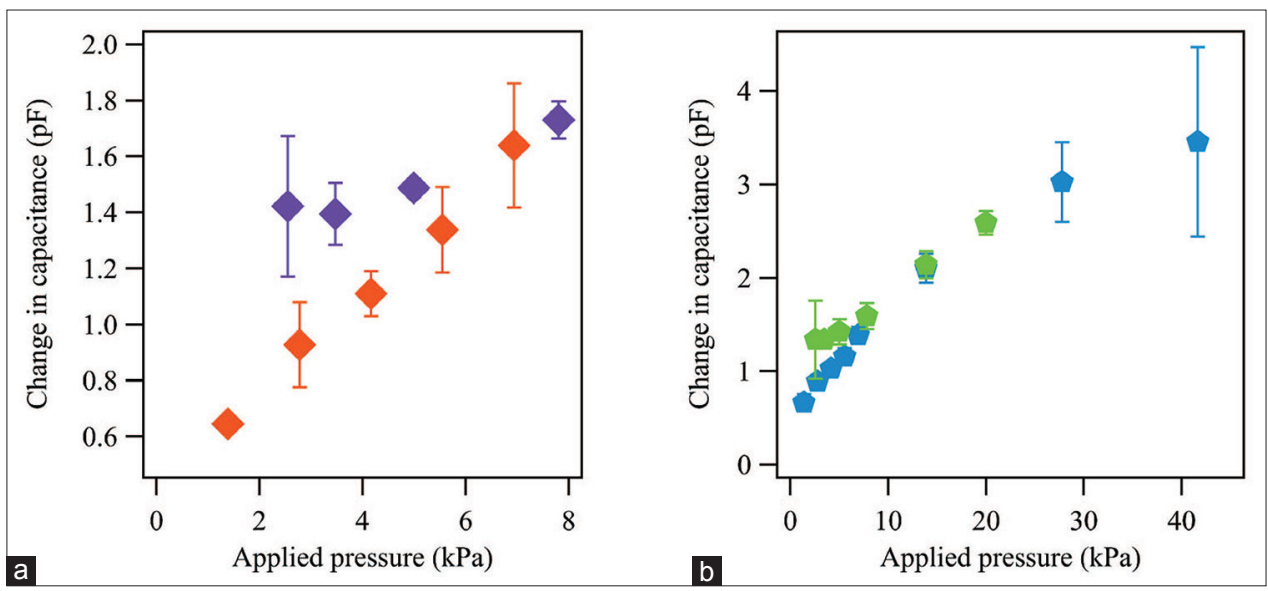

Figure 4: Knitted pressure sensors tested with different pressures. Pressure was changed by either changing the area of the applied force $(\diamond, \bullet)$ or the applied force $(\diamond, \bullet)$. (a) Data collected using the $2.6 \mathrm{~mm}$ thick knitted spacer pressure sensor. The sensor was sensitive to changes pressure up to $7.8 \mathrm{kPa}$. At higher pressure, the sensor did not work. Changes in pressure by changing the applied force $(\diamond)$ or by changing the area over which force was applied ( $\downarrow)$, gave similar, but not identical results. (b) Data collected using the 2.8-mm thick knitted spacer pressure sensor. The sensor was constantly sensitive to changes pressure up to $20 \mathrm{kPa}$. Above this point, the sensor still functioned correctly up to 41.6 $\mathrm{kPa}$ when force was applied over a $7.1 \times 10^{-4} \mathrm{~m}^{2}$ area, however gave inconsistent results when a $9.8 \mathrm{~N}$ of force was applied over areas of $3.1 \times 10^{-4} \mathrm{~m}^{2}$ and $1.8 \times 10^{-4} \mathrm{~m}^{2}$ (corresponding to 31.2 and $55.5 \mathrm{kPa}$, respectively). Changes in pressure by changing the applied force ( $\bullet$ ) or changes in the area over which force was applied $(\bullet)$ were in agreement with one another within the experimental error

below in Table 2. The maximum pressure that the sensors consistently operated up to have been presented for both of the testing methods used in this work. Change in capacitance values at 6.9 and $7.8 \mathrm{kPa}$, for experiments conducted where the applied force or the area of applied force was changed, respectively, is also tabulated to demonstrate the level of sensitivity of each sensor design.

The data show a clear increase in sensitivity when a thinner pressure sensor is used; however, the thinner sensors were not able to operate at higher pressures.

\section{CONCLUSIONS}

This work has considered the response of two different textile pressure sensors, with pressure monitoring for wheelchair users being the targeted application. Three major factors have been considered and explored such as the force response of the sensor, the area of applied force response of the sensor, and the hysteresis of the sensor. In addition, experiments were conducted to optimize the sensors' thickness.

Two sensor designs were investigated: a knitted pocket pressure sensor which used two layers of fabric with knitted silver electrodes in which spacer materials could be inserted and a knitted spacer pressure sensor, where a knitted 3D spacer fabric with knitted silver electrodes on either face of the fabric was produced as a single fabric layer. The former was similar to other sensors previously described in the literature. The study showed that the knitted spacer pressure sensor was not pressure sensitive; instead, its response was dictated by a complex combination of the force applied and area that the force was applied over.

Conversely, the knitted spacer pressure sensor operated up to $25 \mathrm{kPa}$, which was the maximum pressure of interest. At higher pressures, measurement errors were incurred for absolute pressure measurement $(\sim 20 \%$ at $\sim 30 \mathrm{kPa})$; however, the general trend of an increase in pressure leading to an increase in the change in capacitance was still observed. This would be able to provide a suitable indicator of the application of pressure to the sensor.

The effects of hysteresis were also examined observing that the knitted pocket pressure sensor was subject to hysteretic effects, while the hysteresis was less significant for the knitted spacer pressure sensor. This was likely due to the single-layer structure of the knitted spacer pressure sensor, which offers a distinct advantage over the other design.

The thickness of the knitted spacer pressure sensor was optimized by exploring three different thicknesses. It was observed that the two thinner sensors $(2.6 \mathrm{~mm}, 2.8 \mathrm{~mm})$ could not consistently measure pressures up to $25 \mathrm{kPa}$. It was therefore determined that the 3.2-mm thick knitted spacer pressure sensor was the most suitable design tested.

Ultimately, this work has characterized two designs of textile sensor. The research has shown that the 
Hughes-Riley, et al.: A knitted spacer pressure sensor

Table 2: Summary of operational limitations of the pressure sensor

\begin{tabular}{|c|c|c|c|c|}
\hline & $\begin{array}{c}\text { Upper limit of } \\
\text { operation: Fixed force } \\
\text { experiments }(\mathrm{kPa})\end{array}$ & $\begin{array}{c}\text { Upper limit of } \\
\text { operation: Fixed area } \\
\text { experiments }(\mathrm{kPa})\end{array}$ & $\begin{array}{l}\text { Change in capacitance } \\
\text { at } 7.8 \mathrm{kPa} \text { for fixed force } \\
\text { measurements (pF) }\end{array}$ & $\begin{array}{c}\text { Change in capacitance } \\
\text { at } 6.9 \mathrm{kPa} \text { for fixed area } \\
\text { measurements (pF) }\end{array}$ \\
\hline Knitted pocket pressure sensor & $\begin{array}{c}55.5 \text { (not pressure } \\
\text { sensitive) }\end{array}$ & $\begin{array}{c}41.6 \text { (not pressure } \\
\text { sensitive) }\end{array}$ & $0.8 \pm 0.2$ & $0.5 \pm 0.1$ \\
\hline Knitted spacer pressure sensor & 55.5 & 41.6 & $1.2 \pm 0.1$ & $0.9 \pm 0.2$ \\
\hline Knitted spacer pressure sensor (2.6 mm) & 7.8 & 6.9 & $1.7 \pm 0.1$ & $1.6 \pm 0.2$ \\
\hline Knitted spacer pressure sensor (2.8 mm) & 20.0 & 41.6 & $1.6 \pm 0.1$ & $1.4 \pm 0.1$ \\
\hline
\end{tabular}

knitted pocket pressure sensor did not act as a true pressure sensor in the pressure range of interest. A novel knitted spacer pressure sensor was developed and characterized, which was shown to be pressure sensitive up to $25 \mathrm{kPa}$.

Future work will scale up the prototype sample to create a wheelchair cushion cover and design supporting electronics. This will necessitate a further characterization of the chosen knitted pressure sensor exploring the pressure sensitivity at smaller pressure increments than presented in this work. The step-response time of the sensor should also be investigated, although it is envisioned that the final system will operate under mostly static conditions, requiring measurements every 1-2 min; higher frequency data collection may be necessary for other applications. As this sensor has been shown to be pressure sensitive in the $0-8 \mathrm{kPa}$ pressure range, further investigations will explore creating a mattress cover for pressure monitoring for patients prone to formation of ulcers (bed sores) in hospitals.

\section{Acknowledgment}

The authors would like to thank Jim Boxall for taking the photograph of the knitted spacer pressure sensor shown in Figure 1b.

\section{Financial support and sponsorship}

The authors would like to acknowledge Nottingham Trent University for providing financial support to conduct this research. This research was also partially funded through the European Regional Development Fund Enabling Innovation project.

\section{Conflicts of interest}

There are no conflicts of interest.

\section{REFERENCES}

1. Bennett G, Dealey C, Posnett J. The cost of pressure ulcers in the UK.
Age Ageing 2004;33:230-5.

2. Stockton L, Parker D. Pressure relief behaviour and the prevention of pressure ulcers in wheelchair users in the community. J Tissue Viability 2002;12:84, 88-90, 92.

3. Ferguson-Pell M, Cardi MD. Prototype development and comparative evaluation of wheelchair pressure mapping system. Assist Technol 1993;5:78-91.

4. Gyi DE, Porter JM, Robertson NK. Seat pressure measurement technologies: Considerations for their evaluation. Appl Ergon 1998;29:85-91.

5. Axisa F, Schmitt PM, Gehin C, Delhomme G, McAdams E, Dittmar A, et al. Flexible technologies and smart clothing for citizen medicine, home healthcare, and disease prevention. IEEE Trans Inf Technol Biomed 2005;9:325-36.

6. Sergio M, Manaresi N, Tartagni M, Guerrieri R, Canegallo R. A textile based capacitive pressure sensor. Proc IEEE Sens 2002;2:1625-30

7. Ashruf CM. Thin flexible pressure sensors. Sens Rev 2002;22:322-7.

8. Meyer J, Lukowicz P, Troster G. Textile Pressure Sensor for Muscle Activity and Motion Detection. $10^{\text {th }}$ IEEE International Symposium on Wearable Computers; 2006. p. 69-72.

9. Holleczek T, Rüegg A, Harms H, Tröster G. Textile pressure sensors for sports applications. IEEE Sensors 2010;2010. p. 732-7.

10. Mannsfeld SC, Tee BC, Stoltenberg RM, Chen CV, Barman S, Muir BV, et al. Highly sensitive flexible pressure sensors with microstructured rubber dielectric layers. Nat Mater 2010;9:859-64.

11. Meyer J, Arnrich B, Schumm J, Troster G. Design and modeling of a textile pressure sensor for sitting posture classification. IEEE Sens J 2010;10:1391-8.

12. Zhang RQ, Li JQ, Li DJ, Xu JJ. Study of the structural design and capacitance characteristics of fabric sensor. Adv Mater Res 2011;194:1489-95.

13. Castano LM, Flatau AB. Smart fabric sensors and e-textile technologies: A review. Smart Mater Struct 2014;23:053001.

14. Hughes-Riley $\mathrm{T}, \mathrm{Dias} \mathrm{T}, \mathrm{Cork} \mathrm{C}$. A historical review of the development of electronic textiles. Fibers 2018;6:34.

15. Hoffmann T, Eilebrecht B, Leonhardt S. Respiratory monitoring system on the basis of capacitive textile force sensors. IEEE Sens J 2011;11:1112-9.

16. Ferguson-Pell MW. Design criteria for the measurement of pressure at body/support interfaces. Eng Med 1980;9:209-14.

17. Hobson DA. Comparative effects of posture on pressure and shear at the body-seat interface. J Rehabil Res Dev 1992;29:21-31.

18. Gerhardt LC, Mattle N, Schrade GU, Spencer ND, Derler S. Study of skin-fabric interactions of relevance to decubitus: Friction and contact-pressure measurements. Skin Res Technol 2008;14:77-88. 